\title{
A contextual interference account of distinctiveness effects in recognition
}

\author{
HEEKYEONG PARK \\ Carnegie Mellon University, Pittsburgh, Pennsylvania \\ JASON ARNDT \\ Middlebury College, Middlebury, Vermont \\ and \\ LYNNE M. REDER \\ Carnegie Mellon University, Pittsburgh, Pennsylvania
}

\begin{abstract}
In this article, we report on two experiments that aimed to shed light on the memorability effect that derives from varying the uniqueness of contextual cues presented at encoding and retrieval. We sought to understand the locus of the recognition advantage for studying and testing words with nominally irrelevant features that are rarely shared with other words ("low-fan" features) as compared with features that are studied with more words ("high-fan" features). Each word was studied with one high-fan feature and one low-fan feature, but only one of the two features was reinstated at test. Recognition judgments were more accurate when the low-fan feature was reinstated than when the high-fan feature was reinstated. The data suggest that encoding cues that suffer from contextual interference negatively affect retrieval and do so by hindering recollection-based processing.
\end{abstract}

Several recent studies have found that memory for words is affected not only by whether the font studied during encoding is reinstated at test (Graf \& Ryan, 1990), but also by the number of other words that share the font (Diana, Peterson, \& Reder, 2004; Reder, Donavos, \& Erickson, 2002). This effect has been called the font fan effect, because the postulated memory representations vary in the number of contextual associations that "fan" out from the node that represents a particular font (see Figure 1). The mechanistic account of this result proposed by Reder et al. (2002) assumes that the amount of activation that spreads from the font source to the node representing the encoding event varies as a function of the fan or number of competitors that share the activation. Further details about the font fan effect on memory will be discussed later. The important issue to understand at this juncture is that these memory effects have been assumed to result from retrieval processes.

Alternatively, it is possible that the font fan effect reflects an effect of distinctiveness, whereby participants pay more attention to distinctive fonts (i.e., those that were shown fewer times during encoding). It has been well es-

H.P. is now at the University of California, Irvine. This work was supported by Grant 2-R01-MH52808 from the National Institute of Mental Health to L.M.R. We thank Sandi Gani, Beth Newton, and Margaret Peterson for assistance with data collection. Parts of this research were reported at the 44th annual meeting of the Psychonomic Society, Vancouver, Canada, November 2003. Correspondence should be addressed to H. Park, Center for the Neurobiology of Learning and Memory, University of California, Irvine, CA 92697-3800 (e-mail: hkpark@uci.edu). tablished in the memory literature that distinctive stimuli are better remembered than nondistinctive stimuli, and, conceivably, the effect of "contextual fan" of the features that we manipulate might well be only an expression of this distinctiveness. For example, it has been shown that both semantically distinctive words (e.g., Hunt \& Mitchell, 1982; Rajaram, 1998; Schmidt, 1985) and perceptually distinctive items (e.g., Hunt \& Elliott, 1980; Hunt \& Mitchell, 1982; Rajaram, 1998; Zechmeister, 1972) improve recognition memory.

Prior theorists have suggested two basic types of distinctiveness effects: those due to primary distinctiveness and those due to secondary distinctiveness (Schmidt, 1991). Primary distinctiveness effects occur when the properties of an item deviate from the properties of other items in a given study list. Such distinctiveness effects have been demonstrated by isolating physical features, such as font size or color of an item, from other items in a list (e.g., Fabiani \& Donchin, 1995; Kishiyama \& Yonelinas, 2003) or by isolating an item from other items in a list via membership in a semantic category (Fabiani \& Donchin, 1995; Geraci \& Rajaram, 2004; Hunt \& Lamb, 2001; Schmidt, 1985; von Restorff, 1933). In these cases, the isolating feature was contrasted with other homogeneous features in the experimental context. Secondary distinctiveness, on the other hand, occurs when the properties of an item deviate from the properties of items in one's semantic memory, or long-term store. Thus, this type of distinctiveness effect occurs when deviance can be defined in terms of dissimilarity of an inherent characteristic of an item relative to the characteristics of a class of items or a particular 


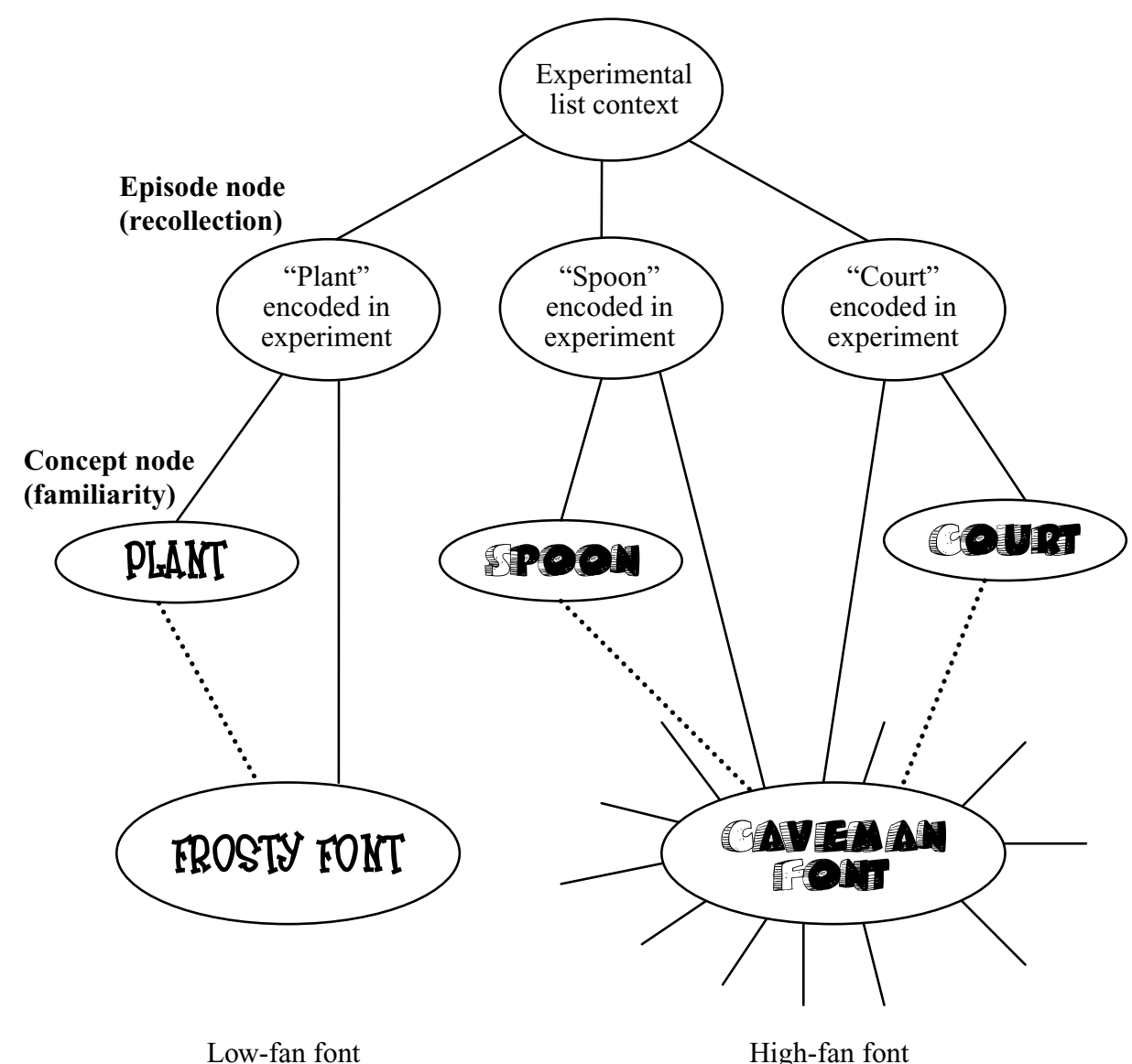

Figure 1. An illustration of the representation of memory traces for words presented in unusual fonts that were seen with different numbers of other words.

study list. An example of this type of distinctiveness effect is the memory advantage of orthographically uncommon/ exceptional words over regular words (Hirshman \& Jackson, 1997; Hunt \& Elliott, 1980; Rajaram, 1998). Given this characterization of distinctiveness effects, font fan effects may be thought of as an instance of primary distinctiveness.

One central question in the literature on distinctiveness effects is the extent to which the memory advantage for distinctive items derives from encoding-based processes (e.g., salience or enhanced attention; Fabiani \& Donchin, 1995) or retrieval-based processes (e.g., item-specific processing used to access details of an encoding episode; Hunt \& McDaniel, 1993). Whereas the literature on distinctiveness effects in recall has generally suggested that retrieval processes must play at least some role (Schmidt, 1991), the literature on distinctiveness effects in recognition memory has been less clear on the mechanisms involved.

As noted above, Reder et al. (2002) attributed fan effects, and by association, font distinctiveness effects, to retrievalbased processes. Hunt and his colleagues (Dunlosky, Hunt, \& Clark, 2000; Hunt, 2003; Hunt \& Lamb, 2001; Hunt \& McDaniel, 1993; Hunt \& Smith, 1996; Smith \& Hunt, 2000) have consistently argued that distinctiveness func- tions to separate items in memory from one another at retrieval. Further, Hunt (2003) has recently argued that distinctiveness can play a role both to enhance recollection of studied items as well as to reject familiar, unstudied items. Rajaram (1998) argued that distinctiveness is critical to the experience of recollection phenomenology, whereby items that contain perceptually or conceptually distinctive features give rise to the experience of recollection. Thus, these three perspectives would all argue that font fan effects are a product of retrieval-based processes. On the other hand, Kishiyama and Yonelinas (2003) have suggested that distinctiveness influences recognition memory due to processes occurring at both encoding and retrieval. In particular, Kishiyama and Yonelinas argue that distinctiveness influences both recollection- and familiarity-based recognition memory. Further, these authors argue that the effect of distinctiveness on recollection is produced by encoding-based factors, whereas the effect of distinctiveness on familiarity is produced by retrieval-based factors. Finally, many standard views of distinctiveness effects largely attribute the memorial advantage of distinctiveness to encoding-based processes, such as enhanced attention (e.g., Jenkins \& Postman, 1948) or salience (e.g., Green, 1956; see Schmidt, 1991, for a review). 
We now turn to a description of the theoretical framework that inspired these experiments. Although the central claim of this theory - that retrieval-based processes produce font fan effects - is also a property of other theories of distinctiveness (Hunt \& McDaniel, 1993; Rajaram, 1998), the framework that was used to conceive of these studies possesses an explicit mechanism for producing font fan effects: contextual competition. In contrast, other retrieval-based theories of distinctiveness effects in recognition do not provide as detailed an account of the processes at work in producing font fan effects. As we will show below, the notion of contextual competition provides several detailed predictions that are tested in our studies. Thus, we highlight the account outlined by Reder and colleagues (2002) as an organizing framework for thinking about the font fan effect. The point is to argue not that contextual competition is the only explanation, but rather that it provides several novel predictions that are amenable to testing, which we explore in these studies.

\section{Brief Description of the Source of Activation Confusion Model for Recognition Memory}

As illustrated in Figure 1, the basic memory representation that is assumed by the source of activation confusion (SAC) model to account for recognition performance involves two types of memory representations: concept nodes and episode nodes (e.g., Reder et al., 2002; Reder et al., 2000). ${ }^{1}$ Concept nodes encapsulate the history of experience with any particular word, such as its meaning(s), the number of prior exposures to that word, and the recency of those exposures. Episode nodes encapsulate the details of a particular encounter with a word, such as the room context, the experimenter, the feelings that the participant had at the time of the experiment, and so forth. When a word is studied in an experiment, the information that is bound to the episode node comes from the concept node and from the experimental context. The latter information can be represented as a single node when all the features of the context are thought to be the same across all studied items. When aspects of the study context, such as visual features of the studied words (e.g., fonts) vary from item to item, then those features will be bound separately to the episode node because they are not general aspects of the study context.

On a recognition test, participants are assumed to make decisions based on one of two processes: recollection or familiarity. Recollection judgments are based on the ability to retrieve the episode node, whereas familiarity judgments are usually based on the level of strength of the concept node and rarely (spuriously) on the level of activation of the font node (Diana et al., 2004). The presentation of a word on a recognition memory test activates that word's concept representation, causing it to spread activation across its associations. Further, the presentation characteristics of a word on a recognition test are also activated, such that a font's representation spreads activation across its associations (e.g., Arndt \& Reder, 2003; Reder et al., 2002). If the font that is used at test matches the font encoded with the word during study, then the font that is activated will have a link to the episode node representing the presentation of that word in the study list. The link between the font node and the episode node will allow for activation to spread from the font to the episode node, thereby increasing the activation level of the episode node. If the activation that accrues at an episode node is sufficiently high, that episode will be retrieved, and the participant will recollect that word's presentation in the experiment. On the other hand, if memory search is unsuccessful in retrieval of the episode node, a person may still make a positive recognition judgment if the concept node is sufficiently active. However, recognition judgments based on the concept node are familiarity based. Font features can also be used as a source of familiarity when font fan has its effect on false alarms, but it does not always happen (Diana et al., 2004).

The retrieval explanation for font fan effects within the SAC model is based on the notion that words studied in low-fan fonts will receive more activation from the representation of the font in which they were presented during study if that font is again used to present the words at test. Further, the recognition of words presented in low-fan fonts should improve due to improved ability to retrieve episode nodes, because font representations are associated with the episode nodes of items presented at study. Thus, the SAC model posits that the influence of font fan on recognition memory should produce differences in recollection and not differences in familiarity. The mechanism that produces differential activation spread to words presented in low-fan fonts (when compared with words presented in high-fan fonts) is contextual competition, implemented in the model as the number of associations emanating from a font's representation. Thus, a font that is studied with a single word in the study list will be a better retrieval cue than a font that is studied with many words in a study list, because in the latter case, the font will distribute its activation at test across a much larger array of associations than in the former.

In the present article, we seek to add to the extant literature in two ways. First, we seek to further test the extent to which font fan effects are driven by encoding- or retrievalbased processes with two experiments that hold the perceptual distinctiveness of study items constant, while varying the distinctiveness of perceptual features reinstated at retrieval in a recognition memory task. ${ }^{2}$ Second, we seek to examine the extent to which the memorial advantage of presenting test words in distinctive fonts is associated with recollection- or familiarity-based recognition.

To examine whether the number of contextual associations to a font has a greater effect on retrieval competition at test than a differential influence at encoding, we attempted to equate item processing and overall distinctiveness of study items. This was accomplished by presenting each list item with two features, one distinctive (low fan) and one less distinctive (high fan). At test, only one of the two features was reinstated, either the low-fan or the highfan feature. By equating the distinctiveness of the stimulus during encoding, we could measure the effectiveness of the two types of cues at retrieval and thereby examine 
the SAC retrieval-based account. As outlined above, two theories of the role of distinctiveness in recognition ( $\mathrm{Ra}$ jaram, 1998; Reder et al., 2002) suggest that distinctiveness should have its primary influence on recollectionbased recognition memory. On the other hand, Kishiyama and Yonelinas (2003) have suggested that not all types of distinctiveness influence recollection alone, with some types of distinctiveness influencing both recollection and familiarity. Specifically, Kishiyama and Yonelinas have proposed that the effects of distinctiveness on recollection are a product of processes that occur at encoding, whereas the effects of distinctiveness on familiarity are a product of processes that occur at retrieval.

\section{EXPERIMENT 1}

In this experiment, two perceptual features (color and font) were associated with each word. The goal was to keep the overall distinctiveness of each word constant during encoding by presenting studied words in one high-fan feature (i.e., used to present many other words) and one low-fan feature (i.e., used to present few other words). At test, only one of the two features was reinstated with the word, while the other feature was kept neutral. For example, if font was to be neutral at test, the word would be presented in a common font such as Times Roman; if color was to be neutral at test, the color of the font would be black instead of one of the colors utilized to present items at study.

If perceptual distinctiveness affects the amount of attention allocated during encoding, then we would not expect a difference in recognition performance for words tested with their low-fan versus their high-fan feature, because all words were studied under the same encoding conditions. On the other hand, if the fan manipulation of the encoding features of a word has its memory effect from contextual competition at retrieval, then we should observe a difference in memory performance based on the fan of the reinstated context cue at test. An additional test of the SAC theoretical account is that these retrieval-based effects should come from the recollection component of recognition rather than familiarity-based responding. According to the SAC model, the low-fan test cue should provide more activation to the episode node than the highfan cue, which in turn will increase the likelihood that the episode node will be retrieved. This means that the effect of reinstating the feature at test should have its impact on the recollective processes rather than the familiarity-based processes. This prediction was evaluated by asking participants to provide remember-know judgments (Tulving, 1985). Therefore, according to the SAC model, one would expect the influence of font fan to be realized in both hit rates and remember responses. Thus, this experiment also serves to examine whether font distinctiveness effects are driven by recollection-based processing, as asserted in the SAC model and the distinctiveness-fluency account (Rajaram, 1996, 1998), or whether font distinctiveness effects influence both recollection- and familiarity-based processes (Kishiyama \& Yonelinas, 2003).

\section{Method}

Participants. Twenty-six Carnegie Mellon University students participated in Experiment 1A and 24 participated in Experiment 1B for research experience credit.

Design and Materials. We conducted two versions of the experiment, in which we varied the nature of the perceptual features associated with the study words. During study, each word was presented in an unusual font as one contextual feature. With regard to the other contextual feature, Experiment 1A varied the color of the background on the computer screen, and Experiment $1 \mathrm{~B}$ varied the color of the font. Given that colors have far greater preexperimental exposure than do these atypical fonts, the degree to which varying experimental fan of the color cue would affect performance was unclear. Because of this uncertainty, we instantiated color as a contextual feature two different ways. For expositional purposes, we treat these two different implementations of the color feature as the same manipulation, because the two variants of this manipulation had the same effects on the results. Each color and font was randomly assigned to be either a high-fan or low-fan feature for a given participant. High-fan features (colors or fonts) were shown with 24 different words, whereas low-fan features were shown with 3 different words. Which feature (color or font) was high fan or low fan for any particular word was randomly determined for each participant, with the constraint that each word had one high- and one low-fan feature and that each feature type (color and font) was used equally often as a high- and as a low-fan feature. Words were also randomly assigned to study and test conditions for each participant. Given these constraints, nine different colors and nine different fonts were required to present 48 words at study.

During the recognition memory test, 48 studied words and 48 new words were presented. For studied items, only one feature from the study phase was reinstated and the other feature was neutral. For example, if a color feature was reinstated with an item, the item was presented in a neutral font (Times Roman); if the studied font was reinstated as the test cue, then the word was presented on a white screen using a black font so the color feature at test was neutral. An equal number of words were tested with their low-fan and high-fan features. Additionally, an equal number of words were tested with the font of study presentation reinstated as with the color of study presentation reinstated. Forty-eight new words were also tested with one of the two contextual features, using the same distribution of the features tested for targets. There were two study test cycles, such that there were 96 old words and 96 new words used in the entire experiment.

Procedure. The participants were instructed that they would be presented with a series of words, one at a time, on a computer monitor, and that study was self-paced. The words were presented in different colors (background colors or font colors) as well as different fonts - one feature associated with many other words and the other feature associated with few other words. They were asked to rate the collective appropriateness of each font and color for the meaning of a word at study, using a scale that ranged from 1 (very inappropriate) to 5 (very appropriate).

Following each study list, the participants were given a recognition memory test. The participants were instructed to judge whether or not they had previously studied each probe word. The participants were explicitly instructed that the perceptual features were incidental and did not need to match in order for them to indicate positive recognition of the word. Following Tulving (1985), the participants were asked to discriminate between old judgments for which they could recollect having studied the word earlier ("remember") and those for which they were confident that they had studied the word even though they could not actually recollect studying it ("know"). They were asked to press the key labeled $R$ for remember, to press the $K$ key, for know, and to press $N$, to indicate that the item was not studied earlier in the experiment.

In order to ensure that the participants understood the difference between the remember and know responses, the participants were 
required to give their own examples of each judgment, following the procedure of Gardiner (1988). After they finished the first studytest block, the participants were asked to complete a demographic questionnaire. Following this, the participants repeated one more study-test block.

\section{Results and Discussion}

We analyzed the data separately for Experiments 1A and $1 \mathrm{~B}$ and the pattern of results was the same. An ANOVA that used experiment version (A or B) as a grouping variable did not produce any significant effects (all $F$ s $<1)$. Therefore, we collapsed over the two variants of the experiment for subsequent analyses, all of which were 2 (fan: high vs. low) $\times 2$ (reinstated feature: font vs. color) within-subjects ANOVAs. Table 1 shows the hit and false alarm rates, partitioned into the proportion of remember and know responses, as a function of whether the reinstated feature was the low- or high-fan feature at study. For hits, there was a main effect of fan $[F(1,49)=$ $\left.13.47, M S_{\mathrm{e}}=0.004\right]$, such that there were more hits when a low-fan feature was reinstated at test than when a highfan feature was reinstated. There was also a main effect of which feature was reinstated $\left[F(1,49)=23.21, M S_{\mathrm{e}}=\right.$ $0.01]$, such that there were more hits when fonts were reinstated than when colors were reinstated. The interaction between fan and reinstated feature was not reliable $(F=$ 2.78, $\left.M S_{\mathrm{e}}=0.007\right)$.

As an additional test of the SAC theoretical account, we examined the proportion of remember judgments for the hits, as well as measures of recollection and familiarity. For remember responses, there was a main effect of fan, showing more remember responses to the items for which a low-fan feature was reinstated $[F(1,49)=24.93$, $\left.M S_{\mathrm{e}}=0.012\right]$. There was also a main effect of reinstated feature $\left[F(1,49)=45.85, M S_{\mathrm{e}}=0.018\right]$, such that participants gave more remember responses when a font was re-presented as the test cue. There was an interaction between fan and reinstated feature $\left[F(1,49)=15.62, M S_{\mathrm{e}}=\right.$ $0.012]$. When a low-fan font was reinstated at test, remember responses were greater than when a high-fan font was reinstated at test $\left[F(1,49)=36.52, M S_{\mathrm{e}}=0.013\right]$, but the same result was not observed when color was reinstated at test $\left[F<1, M S_{\mathrm{e}}=0.011\right]$. Given that colors have greater preexperimental exposure than fonts, this may have led to them being less powerful retrieval cues than fonts, a question we address in the next experiment.

When a corrected measure of recollection - that is, $\left[p\left(R_{\mathrm{hit}}\right)-p\left(R_{\mathrm{FA}}\right)\right]$ - was used (Yonelinas, 2001; Yonelinas $\&$ Jacoby, 1995), all patterns of the analyses for remem- ber judgments to old items were replicated. Thus, there was a low-fan advantage $\left[F(1,49)=24.4, M S_{\mathrm{e}}=0.016\right]$, a stronger font feature effect $\left[F(1,49)=29.31, M S_{\mathrm{e}}=\right.$ $0.016]$, and an interaction between fan and reinstated feature $\left[F(1,49)=20.02, M S_{\mathrm{e}}=0.013\right]$. When familiarity was estimated by measuring only those items that were not recollected [i.e., $F=K /(1-R)$, as prescribed by Yonelinas and Jacoby (1995)], neither of the main effects nor the interaction was significant (feature, $F=2.26, M S_{\mathrm{e}}=$ 0.046; fan, $F<1, M S_{\mathrm{e}}=0.041$; feature $\times$ fan, $F<1$, $\left.M S_{\mathrm{e}}=0.046\right)$. We further calculated $d^{\prime}$ using $F$ to directly compare our results to those of Kishiyama and Yonelinas (2003). Here, we failed to find any significant effects on $d^{\prime}(F)$ with the largest $\left[F(1,49)=1.26, M S_{\mathrm{e}}=0.803\right]$.

For new items, the false alarm rates showed main effects of fan $\left[F(1,49)=3.86, M S_{\mathrm{e}}=0.01\right]$ and reinstated feature $\left[F(1,49)=8.59, M S_{\mathrm{e}}=0.01\right]$, as well as an interaction between fan and reinstated feature $[F(1,49)=5.23$, $\left.M S_{\mathrm{e}}=0.006\right]$. The main effects indicate that there were more false alarms when a high-fan feature was reinstated and when a font was reinstated at test, respectively. The interaction indicates that false alarm rates were greater when a high-fan font was reinstated at test $[F(1,49)=$ 7.33, $\left.M S_{\mathrm{e}}=0.01\right]$, but not when a high-fan color was reinstated $\left(F<1, M S_{\mathrm{e}}=0.006\right)$. This font fan effect on false alarms is consistent with prior reports, and likely results from spurious familiarity judgments based on the activation level of the font node (Diana et al., 2004). However, analyses of familiarity-based false alarms fail to show a significant main effect or interaction (feature, $F=$ $1.33, M S_{\mathrm{e}}=0.01$; fan, $F=2.44, M S_{\mathrm{e}}=0.009$; feature $\times$ fan, $\left.F=3.71, M S_{\mathrm{e}}=0.005\right)$. Since the distinctiveness of all stimuli was held constant during study (each word had one high-fan and one-low fan feature during encoding), encoding time was not expected to vary systematically. In fact, study time did not differ as a function of the fan feature (color high fan, 820.72; font high fan, 804.17; $F<$ $\left.1, M S_{\mathrm{e}}=17,805.72\right)$. Note that when a feature was used as a high-fan feature of an item, the other feature was used as the low-fan feature of the same item.

\section{EXPERIMENT 2}

Although the pattern obtained in Experiment 1 was generally consistent with the predictions of the SAC model, the effect of font fan was larger than the effect of color fan. We suspect that this is because color is a more common preexperimental feature, and therefore the ma-

Table 1

Proportions of Remember $(R)$ and Know $(K)$ Responses and Familiarity Estimates $(F)$ for Hits and False Alarms (FAs) as a Function of the Fan of Reinstated Feature in Experiment 1

\begin{tabular}{|c|c|c|c|c|c|c|c|c|c|c|c|c|}
\hline \multirow[b]{3}{*}{ Response } & \multicolumn{6}{|c|}{ Font Reinstated } & \multicolumn{6}{|c|}{ Color Reinstated } \\
\hline & \multicolumn{3}{|c|}{ Low Fan } & \multicolumn{3}{|c|}{ High Fan } & \multicolumn{3}{|c|}{ Low Fan } & \multicolumn{3}{|c|}{ High Fan } \\
\hline & $R$ & $K$ & $F$ & $R$ & $K$ & $F$ & $R$ & $K$ & $F$ & $R$ & $K$ & $F$ \\
\hline Hits & .71 & .19 & .61 & .57 & .27 & .64 & .52 & .29 & .58 & .51 & .29 & .58 \\
\hline FAs & .07 & .14 & .15 & .09 & .17 & .19 & .05 & .15 & .15 & .05 & .15 & .16 \\
\hline
\end{tabular}


nipulation of experimental frequency was insufficient to outweigh the preexperimental exposure history of colors. To test this possibility, we replaced color with a less common contextual cue, voice. Words were aurally presented in different voices at the same time that they were visually presented in different fonts. This experiment employed nine different voices and nine different fonts, just as Experiment 1 used nine different colors and fonts. In Experiment 1 , color or font was made neutral at test, whereas in this experiment, only one of the two encoding features was reinstated at test.

\section{Method}

Participants. Twenty-one Carnegie Mellon University students participated in Experiment 2, either to satisfy a research experience requirement or in exchange for $\$ 7$ payment

Design and Materials. Nine different voices (5 male and 4 female) and nine unusual fonts were used to present the words aurally and visually during study. Assignment of voices and fonts to fan condition was randomly determined for each participant as was assignment of fonts and voices to words. In all, 48 words were presented at study, each word presented with two features, one low fan (shared with 3 words) and one high fan (shared with 24 words). Only one of the two encoding features was presented at test, either the voice or the font. Likewise, the 48 new words used as foils were either only heard or only read at test, always with one of the voices or fonts used with the studied items. Half of these foils were presented aurally and half visually, just like the target probes, using the same distribution of high- and low-fan features that was used for studied items on the recognition test.

Procedure. The procedure of Experiment 2 was very similar to that of Experiment 1 except that the participants heard the words through headphones in addition to viewing them on a computer screen. The study phase involved presenting words, one at a time, in two modalities simultaneously: visually, using an unusual font on the monitor, and aurally, with one of a set of unfamiliar voices heard through headphones. Unlike in Experiment 1, study time was not self-paced. Rather, the duration of the visual presentation of an item was set to synchronize with the duration of the aural presentation of the item. The participants were asked to rate the combined appropriateness of a font and a voice for the meaning of that particular study word. At test, the word was presented either aurally (with a blank screen) or visually (with no voice). When the probe was presented, the participants were asked to indicate its studied status with one of three judgments: remember, know, or new.

\section{Results and Discussion}

The results of Experiment 2, shown in Table 2, are similar to the results of Experiment 1. All dependent measures were analyzed with 2 (fan: high vs. low) $\times 2$ (reinstated feature: font vs. voice) within-subjects ANOVAs. Hits were higher when the test item was presented in a low-fan feature $\left[F(1,20)=7.13, M S_{\mathrm{e}}=0.012\right]$, as well as when the feature reinstated at test was a font $[F(1,20)=22.47$, $\left.M S_{\mathrm{e}}=0.023\right]$. However, fan and reinstated feature did not interact $\left(F<1, M S_{\mathrm{e}}=0.019\right)$.

When hit rates were partitioned into remember and know responses, remember responses showed the same pattern of results as the overall hit rates. That is, participants gave more remember responses when the reinstated feature was a low-fan than when it was a high-fan feature $\left[F(1,20)=11.44, M S_{\mathrm{e}}=0.016\right]$ and when the reinstated feature was a font $\left[F(1,20)=16.98, M S_{\mathrm{e}}=0.065\right]$. Further, the interaction between reinstated feature and font was not reliable $\left(F<1, M S_{\mathrm{e}}=0.027\right)$.

When hit rates were corrected with false alarms for remember responses $\left[p\left(R_{\mathrm{hit}}\right)-p\left(R_{\mathrm{FA}}\right)\right]$, there was an effect of font fan $\left[F(1,20)=9.11, M S_{\mathrm{e}}=0.016\right]$, such that reinstating a low-fan feature produced greater corrected remember responses than reinstating a high-fan feature. There was also a main effect of reinstated feature $\left[F(1,20)=17.31, M S_{\mathrm{e}}=0.042\right]$, such that recollection was higher when a font rather than a voice was the reinstated feature. Finally, the interaction between reinstated feature and font was not reliable $\left(F<1, M S_{\mathrm{e}}=0.034\right)$. Thus, the analyses on a standard measure of recollection replicated all of the results from analyses of remember judgments to old items, and suggest that the hit rate advantage for items when a low-fan feature was reinstated at test was reflected in recollection-based responding. Further, and in contrast with Experiment 1, we found evidence that both perceptual features (voice and font) facilitated memory when they were associated with fewer study items (i.e., when they were assigned to be low-fan features).

Replicating the results found in Experiment 1, the corrected familiarity measure [i.e., $F=K /(1-R)$ ] failed to show an effect of fan, and also did not show an interaction between fan and reinstated feature. In contrast with the results from Experiment 1, the $F$ estimate was found to be greater when the reinstated feature was a font $[F(1,20)=$ 7.49, $\left.M S_{\mathrm{e}}=0.045\right]$. Replicating Experiment 1, analyses of $d^{\prime}(F)$ failed to show a significant main effect or interaction (feature, $F<1, M S_{\mathrm{e}}=0.854$; fan, $F=1.42$, $M S_{\mathrm{e}}=1.25$; feature $\times$ fan, $\left.F<1, M S_{\mathrm{e}}=0.739\right)$. Thus, consistent with Experiment 1, we found evidence that distinctiveness has its effect on a recollection-based process at retrieval, rather than a familiarity-based process.

Finally, there were more false alarms when new words were presented in a font than when they were presented in a voice $\left[F(1,20)=9.09, M S_{\mathrm{e}}=0.033\right]$. Neither the fan main effect nor the interaction between fan and reinstated feature was reliable in the false alarm analyses (fan,

Table 2

Proportions of Remember $(R)$ and Know $(K)$ Responses and Familiarity Estimates $(F)$ for Hits and False Alarms (FAs) as a Function of the Fan of Reinstated Feature in Experiment 2

\begin{tabular}{|c|c|c|c|c|c|c|c|c|c|c|c|c|}
\hline \multirow[b]{3}{*}{ Response } & \multicolumn{6}{|c|}{ Font Reinstated } & \multicolumn{6}{|c|}{ Voice Reinstated } \\
\hline & \multicolumn{3}{|c|}{ Low Fan } & \multicolumn{3}{|c|}{ High Fan } & \multicolumn{3}{|c|}{ Low Fan } & \multicolumn{3}{|c|}{ High Fan } \\
\hline & $R$ & $K$ & $F$ & $R$ & $K$ & $F$ & $R$ & $K$ & $F$ & $R$ & $K$ & $F$ \\
\hline Hits & .58 & .31 & .75 & .48 & .37 & .71 & .35 & .41 & .64 & .26 & .41 & .57 \\
\hline FAs & .07 & .21 & .23 & .06 & .27 & .28 & .03 & .15 & .16 & .03 & .17 & .18 \\
\hline
\end{tabular}


$F<1, M S_{\mathrm{e}}=0.015 ;$ fan $\times$ feature, $\left.F<1, M S_{\mathrm{e}}=0.008\right)$. Analyses of familiarity-based false alarms also showed the same pattern of results: There were more familiaritybased false alarms when new words were presented in a font than when they were presented in a voice $[F(1,20)=$ $\left.7.73, M S_{\mathrm{e}}=0.022\right]$; however, the fan main effect and the interaction failed to reach significance (fan, $F=1.34$, $M S_{\mathrm{e}}=.024 ;$ fan $\times$ feature, $\left.F<1, M S_{\mathrm{e}}=0.01\right)$. Thus, the results of the analyses of false alarms in this experiment contrast with those of Experiment 1, as well as with prior reports (Diana et al., 2004) that fonts with greater exposure sometimes serve as a spurious source of familiarity in recognition memory.

\section{GENERAL DISCUSSION}

In this article, we sought to further examine the bases of perceptual fan effects. Specifically, we tested encodingbased explanations against retrieval-based explanations, finding evidence for retrieval-based explanations (e.g., Rajaram, 1998; Reder et al., 2002). We also assessed whether the recognition advantage for items tested in a low-fan feature was associated with recollection-based processes or familiarity-based processes, and found evidence that the advantage was reflected in changes in recollectionbased processes. We elaborate on the significance of these findings below.

The finding that perceptual distinctiveness effects (see note 2) are produced by recollection-based processes is consistent with several theories of distinctiveness effects on memory (e.g., Hunt \& McDaniel, 1993; Rajaram, 1996, 1998; Reder et al., 2002). All of these theories suggest that the effects of distinctiveness occur at retrieval, and tend to be associated with recollection-based processing. Although there are subtle differences among the theories in the mechanisms giving rise to distinctiveness effects, those differences are not strongly distinguished by the present results. An analysis of these results in terms of the contextual competition mechanism inherent in the SAC model (Reder et al., 2002; Reder et al., 2000), however, is particularly relevant. Whereas the present results may not provide unique support for $\mathrm{SAC}$, in the sense that alternate explanations such as the recollection-fluency perspective of Rajaram $(1996,1998)$ can also account for these results, there are two primary reasons why we find the SAC account of these data to be informative. First, the SAC model provides a formal specification of the mechanisms producing the perceptual distinctiveness effects in these experiments: contextual competition. It is important to note that this mechanism is a fundamental property of the theory, and has been applied not only to explain fan effects, but also to other recognition memory phenomena, such as the word frequency effect (Reder et al., 2000), the list-length effect (Cary \& Reder, 2003), and the liststrength effect (Diana \& Reder, 2005). Second, the SAC model advances the strong prediction that perceptual fan effects should be evident in recollection-based processing, because of the view that details of study presentation are associated with episodic representations. Thus, the SAC model can explain not only why perceptual fan effects occur, but also why these effects are reflected in recollection-based processing, and only when the perceptual feature present at study was reinstated at test (Reder et al., 2002).

Two theories seem to be inconsistent with these results. First, the view that encoding salience (e.g., Green, 1956) or enhanced attention (e.g., Jenkins \& Postman, 1948) plays a role in perceptual fan effects does not seem to be able to explain our results. These encoding-based views suggest that low-fan items should have received extra study processing in the first two experiments, a prediction that was inconsistent with the self-paced study times we measured in Experiment 1. Second, these views suggest that the feature that was reinstated at retrieval should not influence performance, a result not consistent with our data. Although the present results are generally inconsistent with encoding-based views that argue that perceptual fan influences the overall attention to study items, they are not inconsistent with more complex encoding-based views. In particular, a theory that allows attention to be directed to specific subsets of an item's features (e.g., semantics, color, voice, font) may be able to accommodate these results. This view can accommodate the influence of font fan on recognition under the assumption that participants are sensitive to the relative novelty of specific perceptual features at encoding, and that participants therefore encode and store less-common item features with a higher probability than more-common item features. Thus, if one assumes that participants differentially attend to low-fan elements of study items relative to high-fan elements of study items, then when low-fan elements of study items are reinstated at test, those features will produce a stronger match, resulting in the fan effects we observed. Although this theory can explain the results of these experiments, the encoding-based processes that participants need to engage in order to accomplish greater attentional focus to the low-fan dimension of study items seems to be relatively more complex than the alternate offered by the SAC model.

A second theory that also does not appear to provide a comprehensive account of these data is that of Kishiyama and Yonelinas (2003). In their study, although both remember and know responses were greater for distinctive items, the recognition advantage of distinctive items on recollection was found in an intentional encoding condition but not in an incidental encoding condition, whereas familiarity-based judgments were not influenced by the encoding instructions. Their findings were interpreted as evidence that distinctiveness effects on recollection are encoding related, whereas the distinctiveness effects on familiarity are retrieval related. In contrast, our data suggest that distinctiveness has its strongest influence on recollection-based processing, and that perceptually based distinctiveness effects on recollection are due to processes acting at retrieval. What aspects of the two studies could have caused these different conclusions? Conceivably, these differences stemmed from differences in materials and design. For example, Kishiyama and Yonelinas used pictorial stimuli, whereas we used verbal stimuli; their 
lists were much longer than ours; and their presentation rate was very rapid. Longer lists and shorter study presentation times tend to reduce the contribution of recollection and increase the contribution of familiarity to recognition judgments (e.g., Cary \& Reder, 2003; Kinoshita, 1997). Given these trends, it may be reasonable to suggest that the differences between our results and those of Kishiyama and Yonelinas (2003) are in part the result of differences in the extent to which familiarity and recollection played a role in recognition judgments across the two sets of experiments. Further research is needed to resolve the discrepancies among the different studies and delineate the conditions in which familiarity and recollection are influenced by distinctiveness.

One final question concerns the necessity of a dualprocess view for explaining the results of these studies. Although the remember-know paradigm has generally been used as a method to examine recognition memory from a dual-process perspective (Dewhurst \& Conway, 1994; Rajaram, 1998; Tulving, 1985), competing models that rely only on a strength-based familiarity process and the placement of two decision criteria (one for old-new judgments and one for remember judgments) have also been proposed (see, e.g., Donaldson, 1996; Dunn, 2004; Hirshman \& Master, 1997; Wixted \& Stretch, 2004). Although our results do not explicitly prohibit a singleprocess explanation, we believe that the account elaborated throughout this article has properties that are to be favored over standard single-process accounts of rememberknow responses. In particular, as highlighted earlier, the perceptual fan effects observed in these studies were predicted by SAC, including details such as the type of recognition process that should be affected by perceptual fan (recollection) as well as the stage of memory at which fan effects should be observed (retrieval). In contrast, accounting for these results in terms of one of the singleprocess theories of remember-know judgments requires a post hoc explanation that describes the movement of strength distributions for each class of items (a separate distribution for each fan and feature combination) and placement of response criteria. Thus, our preference for the dual-process perspective outlined earlier again relates to its ability to (1) provide specific, testable predictions for these studies, and (2) provide a detailed account of these results in terms of explicit mechanisms that are a fundamental characteristic of the theory (contextual interference). In sum, the present studies support the position that distinctiveness has much of its effect on recollectivebased processing at retrieval.

\section{REFERENCES}

ArNDT, J., \& REDER, L. M. (2003). The effect of distinctive visual information on false recognition. Journal of Memory \& Language, 48, $1-15$.

CARY, M., \& REDER, L. M. (2003). A dual-process account of the listlength and strength-based mirror effects in recognition. Journal of Memory \& Language, 49, 231-248.

Dewhurst, S. A., \& Conway, M. A. (1994). Pictures, images, and recollective experience. Journal of Experimental Psychology: Learning, Memory, \& Cognition, 20, 1088-1098.
Diana, R. A., Peterson, M. J., \& Reder, L. M. (2004). The role of spurious feature familiarity in recognition memory. Psychonomic Bulletin \& Review, 11, 150-156.

DiAnA, R. A., \& REDER, L. M. (2005). The list strength effect: A contextual competition account. Memory \& Cognition, 33, 1289-1302.

DonALDSON, W. (1996). The role of decision processes in remembering and knowing. Memory \& Cognition, 24, 523-533.

Dunlosky, J., Hunt, R. R., \& ClARK, E. (2000). Is perceptual salience needed in explanations of the isolation effect? Journal of Experimental Psychology: Learning, Memory, \& Cognition, 26, 649-657.

Dunn, J. C. (2004). Remember-know: A matter of confidence. Psychological Review, 111, 524-542.

FABIANi, M., \& Donchin, E. (1995). Encoding processes and memory organization: A model of the von Restorff effect. Journal of Experimental Psychology: Learning, Memory, \& Cognition, 21, 224-240.

GARDINER, J. M. (1988). Functional aspects of recollective experience. Memory \& Cognition, 16, 309-313.

Geraci, L., \& Rajaram, S. (2004). The distinctiveness effect in the absence of conscious recollection: Evidence from conceptual priming. Journal of Memory \& Language, 51, 217-230.

Graf, P., \& RyAN, L. (1990). Transfer-appropriate processing for implicit and explicit memory. Journal of Experimental Psychology: Learning, Memory, \& Cognition, 16, 978-992.

Green, R. T. (1956). Surprise as a factor in the von Restorff effect. Journal of Experimental Psychology, 52, 340-344.

Hirshman, E., \& JACKsON, E. (1997). Distinctive perceptual processing and memory. Journal of Memory \& Language, 36, 2-12.

Hirshman, E., \& MASTER, S. (1997). Modeling the conscious correlates of recognition memory: Reflections on the remember-know paradigm. Memory \& Cognition, 25, 345-351.

Hunt, R. R. (2003). Two contributions of distinctive processing to accurate memory. Journal of Memory \& Language, 48, 811-825.

Hunt, R. R., \& Elliott, J. M. (1980). The role of nonsemantic information in memory: Orthographic distinctiveness effects on retention. Journal of Experimental Psychology: General, 109, 49-74.

Hunt, R. R., \& LAмв, C. A. (2001). What causes the isolation effect? Journal of Experimental Psychology: Learning, Memory, \& Cognition, 27, 1359-1366.

Hunt, R. R., \& MCDANIEL, M. A. (1993). The enigma of organization and distinctiveness. Journal of Memory \& Language, 32, 421-445.

Hunt , R. R., \& Mitchell, D. B. (1982). Independent effects of semantic and nonsemantic distinctiveness. Journal of Experimental Psychology: Learning, Memory, \& Cognition, 8, 81-87.

Hunt, R. R., \& Smith, R. E. (1996). Accessing the particular from the general: The power of distinctiveness in the context of organization. Memory \& Cognition, 24, 217-225.

Jenkins, W. O., \& Postman, L. (1948). Isolation and "spread of effect" in serial learning. American Journal of Psychology, 61, 214-221.

Kinoshita, S. (1997). Masked target priming effects on feeling-ofknowing and feeling-of-familiarity judgments. Acta Psychologica, 97, 183-199.

Kishiyama, M. M., \& Yonelinas, A. P. (2003). Novelty effects on recollection and familiarity in recognition memory. Memory \& Cognition, 31, 1045-1051.

RAJARAM, S. (1996). Perceptual effects on remembering: Recollective processes in picture recognition memory. Journal of Experimental Psychology: Learning, Memory, \& Cognition, 22, 365-377.

RAJARAM, S. (1998). The effects of conceptual salience and perceptual distinctiveness on conscious recollection. Psychonomic Bulletin \& Review, 5, 71-78.

REDER, L. M., ARNDT, J., \& PARK, H. (2005). Support for an operational definition of distinctiveness. Unpublished manuscript (www.andrew .cmu.edu/user/reder/unpublished/unpublished.html).

Reder, L. M., Donavos, D. K., \& Erickson, M. A. (2002). Perceptual match effects in direct tests of memory: The role of contextual fan. Memory \& Cognition, 30, 312-323.

Reder, L. M., Nhouyvanisvong, A., Schunn, C. D., Ayers, M. S., Angstadt, P., \& Hiraki, K. (2000). A mechanistic account of the mirror effect for word frequency: A computational model of rememberknow judgments in a continuous recognition paradigm. Journal of Experimental Psychology: Learning, Memory, \& Cognition, 26, 294320. 
SCHMIDT, S. R. (1985). Encoding and retrieval processes in the memory for conceptually distinctive events. Journal of Experimental Psychology: Learning, Memory, \& Cognition, 11, 565-578.

SchmidT, S. R. (1991). Can we have a distinctive theory of memory? Memory \& Cognition, 19, 523-542.

Smith, R. E., \& Hunt, R. R. (2000). The effects of distinctiveness require reinstatement of organization: The importance of intentional memory instructions. Journal of Memory \& Language, 43, 431-446.

Tulving, E. (1985). Memory and consciousness. Canadian Psychology, 26, 1-12.

voN RestorfF, H. (1933). Analyse von Vorgängen im Spurenfeld: 1. Über die Wirkung von Bereichsbildungen im Spurenfeld [Analysis of processes in the trace field: 1 . On the effect of field formations in trace field]. Psychologische Forschung, 18, 299-342.

Wixted, J. T., \& Stretch, V. (2004). In defense of the signal detection interpretation of remember/know judgments. Psychonomic Bulletin \& Review, 11, 616-641.

Yonelinas, A. P. (2001). Consciousness, control, and confidence: The 3 Cs of recognition memory. Journal of Experimental Psychology: General, 130, 361-379.

YonelinAS, A. P., \& JACOBY, L. L. (1995). The relation between remembering and knowing as bases for recognition: Effects of size congruency. Journal of Memory \& Language, 34, 622-643.
ZECHMEISTER, E. B. (1972). Orthographic distinctiveness as a variable in word recognition. American Journal of Psychology, 85, 425-430.

\section{NOTES}

1. Whereas we specifically outline this account for the font fan effect, the general principles should apply to other manipulations in which perceptual components of study presentation have relatively low preexperimental exposure.

2. The above discussion has tacitly assumed that manipulations of font fan affect perceptions of distinctiveness. In fact, we have evidence that it does (Reder, Arndt, \& Park, 2005). Participants were asked to rate the distinctiveness of the fonts after studying these fonts with different words. Fonts that had been seen with many words were subsequently judged as less distinctive even though the words used to present the fonts for ratings were different and even though fonts were randomly assigned to treatment. The effect was not the result of demand characteristic, because the distinctiveness manipulation worked only when participants were rating fonts seen with novel words, not when they were rating the fonts displaying the alphabet.

(Manuscript received February 16, 2005; revision accepted for publication May 10, 2005.) 